\title{
Association of peripheral immunity with cognition, neuroimaging, and Alzheimer's pathology
}

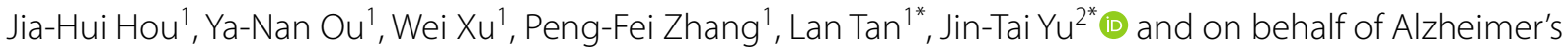

Disease Neuroimaging Initiative

\begin{abstract}
Background: Neuroinflammation has been considered to be a driving force of Alzheimer's disease. However, the association between peripheral immunity and $A D$ has been rarely investigated.
\end{abstract}

Methods: Separate regression analyses were conducted to explore the associations among peripheral immune markers and cognition, neuroimaging, and AD pathology. Causal mediation analyses were used to investigate whether the associations with cognition were mediated by AD pathology.

Results: A total of 1107 participants (43.9\% female, mean age of 73.2 years) from the Alzheimer's Disease Neuroimaging Initiative (ADNI) were included. Regression analyses indicated that elevated neutrophils (NEU) count and neutrophil-lymphocyte ratio (NLR) were associated with lower levels of global cognition, memory function (MEM), and executive function (EF), and reduced brain metabolism by $18 \mathrm{~F}$-fluorodeoxyglucose-positron emission tomography (FDG-PET) as well as greater ventricular volume. An elevated NLR was associated with a lower level of $\beta$-amyloid (AB) and a higher level of total tau (T-tau) in cerebrospinal fluid (CSF), smaller hippocampal volume (HV), and lesser entorhinal cortex (EC) thickness. On the contrary, an elevated level of lymphocytes (LYM) was associated with a higher level of A $\beta$ and a lower level of T-tau in CSF, better cognition, and less atrophy of brain regions (ventricular volume, $H V$, and EC thickness). The associations of LYM and NLR with cognition were mediated by A $\beta$ and T-tau pathology (proportion: 18\% 64\%; $p<0.05$ ).

Conclusions: We revealed that two types of peripheral immune cells (NEU and LYM) and the ratio of these two cell types (NLR) had associations with cognition, neuroimaging, and AD pathology. The associations might be mediated by $A \beta$ and tau pathology.

\footnotetext{
*Correspondence: dr.tanlan@163.com; jintai_yu@fudan.edu.cn

1 Department of Neurology, Qingdao Municipal Hospital, Qingdao University, Qingdao 266071, China

${ }^{2}$ Department of Neurology and Institute of Neurology, Huashan Hospital, Shanghai Medical College, Fudan University, 12th Wulumuqi Zhong Road, Shanghai 200040, China

Data used in preparation of this article were obtained from the Alzheimer's Disease Neuroimaging Initiative (ADNI) database (adni.loni. usc.edu). As such, the investigators within the ADNI contributed to the design and implementation of ADNI and/or provided data but did not participate in analysis or writing of this report. A complete listing of ADNI investigators can be found at: http://adni.loni.usc.edu/wp-content/uploa ds/how_to_apply/ADNI_Acknowledgement_List.pdf.
} permits use, sharing, adaptation, distribution and reproduction in any medium or format, as long as you give appropriate credit to the original author(s) and the source, provide a link to the Creative Commons licence, and indicate if changes were made. The images or other third party material in this article are included in the article's Creative Commons licence, unless indicated otherwise in a credit line to the material. If material is not included in the article's Creative Commons licence and your intended use is not permitted by statutory regulation or exceeds the permitted use, you will need to obtain permission directly from the copyright holder. To view a copy of this licence, visit http://creativecommons.org/licenses/by/4.0/. The Creative Commons Public Domain Dedication waiver (http://creativeco mmons.org/publicdomain/zero/1.0/) applies to the data made available in this article, unless otherwise stated in a credit line to the data. 
Keywords: Peripheral immunity, Alzheimer's disease, Neutrophil-lymphocyte ratio, Neutrophil, Lymphocyte, Mediation

\section{Background}

Alzheimer's disease (AD) is a common neurodegenerative disease pathologically characterized by neurofibrillary tangles and widespread senile plaques. The clinical hallmark of AD is gradual declines in memory and other cognitive functions. Drugs and other interventions are almost impossible to achieve satisfactory efficacy for advanced AD (cognitive impairment) [1]. Therefore, early detection and treatment of $\mathrm{AD}$ are important. The detection methods for existing wellestablished biomarkers, including $\beta$-amyloid $(A \beta)$, phosphorylated-tau (P-tau), and total tau (T-tau) in cerebrospinal fluid (CSF), and techniques such as structural magnetic resonance imaging (MRI) and positron emission tomography imaging for $\mathrm{AD}$ are invasive, expensive, inconvenient, and difficult to implement under resource-limited settings [2]. To reduce its cost and enhance its generalizability, the identification of less-invasive and cheap indicators for AD is becoming increasingly important. Undoubtedly, peripheral blood indicators are a better choice. Furthermore, antiinflammatory therapy might become a new direction for the treatment or prevention of dementia, even during the early stage (absence of cognitive impairment) of AD. Therefore, there is an urgent need to explore the associations of peripheral immunity with cognition, neuroimaging and AD pathology.

It has been proposed that neuroinflammation is a possible cause or driving force of $\mathrm{AD}$ by contributing to neurodegeneration and pathogenesis across all stages of the disease [3]. AD is a systemic disease that involves a dynamic peripheral and central immune responses [4], and growing studies have shown a pivotal contribution of the peripheral immune system. Previous studies have reported changes in the peripheral immune systems of AD patients, especially in aspects of the cell count of neutrophils (NEU) and lymphocytes (LYM) as well as neutrophil-lymphocyte ratio (NLR). As the most important component in the peripheral immune system, NEU were found to co-exist with $A \beta$ deposition in the brain tissues of AD patients [5]. Adaptive immune cells including $\mathrm{T}$ and $\mathrm{B}$ lymphocytes also have a great influence on the inflammatory responses in the brains of AD patients [6]. NLR is a useful and cost-effective biomarker that indicates peripheral systemic inflammation [7]. Existing evidence suggests that peripheral immunity may play a pivotal part in the progression of $\mathrm{AD}$ [8]. However, studies exploring associations between peripheral immunity with $\mathrm{AD}$ in human cohorts are scarce. Herein, we explored the associations between peripheral immune markers (NEU, LYM, and NLR) and cognition, neuroimaging and AD pathology, and tried to determine whether the associations between peripheral immune markers and cognition were mediated by AD core pathology.

\section{Materials and methods \\ Participants}

Data applied in this study were acquired from the ADNI database (http://adniloni.usc.edu), which is designed to test biochemical, clinical biomarkers, genetics, and imaging of AD. Participants received systematic neuropsychological evaluations, as well as neurological and physical examinations at baseline and follow-up, and were offered biological samples such as CSF, blood, and urine throughout the study. This multisite longitudinal biomarker research program authorized by the institutional review committee at all participating locations has acquired written informed consent from participants. The study population is composed of all cognitively normal $(\mathrm{CN})$, mild cognitive impairment (MCI), and $\mathrm{AD}$ participants with available NEU count, LYM count, NLR level, as well as data on cognition, neuroimaging, and $\mathrm{AD}$ pathology.

\section{Peripheral immune cells}

Peripheral immune cells were examined in a subset of participants from ADNI-1. Blood samples were drawn by trained professionals from the venous blood in the morning after an overnight fast, and were sent for analysis on the same day. NEU count, LYM count, and NLR were analyzed using an automated system. More method details could be found at http://adni.loni.usc.edu.

\section{CSF measurements}

CSF was sampled by lumbar puncture, with CSF A $\beta$, CSF T-tau, and CSF P-tau measured at the ADNI Biomarker Core Laboratory (University of Pennsylvania) using a complex platform (xMAP; Luminex Corporation) with Innogenetics (INNO-BIA AlzBio3; Ghent, Belgium; for research use only reagents) immune assay kit-based reagents and analyzed on an automatic Elecsys cobas e 601 instrument (F. Hoffmann-La Roche) by an advanced technology known as electrochemiluminescence immunoassays (Elecsys; Roche Diagnostics, F. Hoffmann-La Roche, Basel, Switzerland). 


\section{Cognition}

Cognitive functions were assessed by several scales. Precisely, global cognition was evaluated by the Mini-Mental State Examination (MMSE), the Alzheimer's Disease Assessment Scale (ADAS), and the Clinical Dementia Rating Sum of Boxes (CDRSB). And cognitive domains were evaluated by inspecting the neuropsychological batteries to confirm elements that can be indicators considered of memory function (MEM) and executive function (EF) $[9,10]$. All assessments were carried out at baseline and follow-up.

\section{Neuroimaging}

The 18F-fluorodeoxyglucose-positron emission tomography (FDG-PET) data were obtained and rebuilt following a standardized process (http://adni.loni.ucla.edu/). Spatial normalization of every participant's positron emission tomography image to the standard template was implemented applying SPM529. We averaged the FDGPET counts in temporal, angular, and posterior cingulate regions. Structural brain images were obtained using a 1.5-T MRI imaging system with T1-weighted MRI scans using a sagittal volumetric magnetization-prepared rapid acquisition gradient-echo sequence. Cortical thickness and subcortical volumes were quantified by a software program (https://surfer.nmr.mgh.harvard.edu/).

\section{Statistical analysis}

On the basis of the cut-off threshold of CSF A $\beta$, the population was divided into $A \beta+$ (concentration levels $\leq$ $192 \mathrm{pg} / \mathrm{ml})$ and $A \beta-(>192 \mathrm{pg} / \mathrm{ml})$ groups. This further resulted in five combinations of biomarkers, consisting of $\mathrm{CN} \mathrm{A} \beta-(\mathrm{CN}-), \mathrm{CN} \mathrm{A} \beta+(\mathrm{CN}+), \mathrm{MCI} \mathrm{A} \beta-(\mathrm{MCI}-)$, $\mathrm{MCI} A \beta+(\mathrm{MCI}+)$, and $\mathrm{AD} A \beta+(\mathrm{AD}+)$ after excluding $15 \mathrm{AD} A \beta-(\mathrm{AD}-)$ subjects [11]. Chi-square analysis and non-parametric analysis were used to test the intergroup differences. Categorical variables were expressed as number (percentage), and continuous variables were expressed as mean \pm standard deviation (SD).

First, we separately assessed the associations of various peripheral immune markers (independent variables) with AD pathology (CSF biomarkers), cognition (global cognition, as well as MEM and EF), and neuroimaging (brain structure and FDG-PET) using multiple linear regression models and linear mixed-effects models. Extreme values of more than 3 SDs from the mean were excluded before the independent variables were normalized by log-transformation. Moreover, we added the interaction analysis between peripheral immune markers with $A P O E$ $\varepsilon 4$ status and gender into multiple linear regression models, to determine whether the associations of peripheral immunity with $\mathrm{AD}$ pathology, cognition, and neuroimaging independent of $A P O E \varepsilon 4$ status and gender as modifiers, and to determine whether strata effects existed.

Next, the mediation analyses were conducted via "mediate," "car," and "lm" packages in R software (version 4.0.3) to explore whether the associations between peripheral immune markers and cognition were mediated by $\mathrm{AD}$ core pathology. Linear regression models were fitted on the basis of the methods proposed by Baron and Kenny [12]. The first equation showed the effect of the independent variable on mediators. The second equation showed the effect of mediators on dependent variables after controlling the influence of independent variables. The third equation showed the total effect of independent variables on dependent variables, the direct effect of independent variables on dependent variables after controlling the influence of mediators, and the indirect effect of independent variables on dependent variables without controlling the influence of mediators.

Covariates in all the correlation analyses included gender, age, $A P O E$ \&4 status, and education level, and intracranial volume was added as a covariate when dependent variables were associated with brain structure. A twotailed $p<0.05$ was considered significant. The $\mathrm{R}$ software (version 4.0.3), GraphPad Prism version 7.00 (San Diego, CA), and IBM SPSS Statistics 26 were applied for figure preparation and statistical analyses.

\section{Results}

\section{Characteristics of participants}

The present analysis included 1107 participants, consisting of $168 \mathrm{CN}-, 123 \mathrm{CN}+, 217 \mathrm{MCI}-, 403 \mathrm{MCI}+$, and $196 \mathrm{AD}+$ participants. The whole population had a female proportion of $43.9 \%$, an age range from 54 to 91 years old (73.20 \pm 7.29 years old), and an APOE $\varepsilon 4$ positive percentage of 47.97\% (Table 1). Among them, 1088 participants were followed up with longitudinal data (see Additional file 1). Except for gender, basic demographics, biomarker levels, cognitive scores, brain structure, and levels of peripheral immune markers all illustrated statistically significant intergroup differences $(p<0.05)$.

As shown in Fig. 1, baseline peripheral NEU count was significantly higher in the AD+ group than in the MCI$(p<0.001)$, and $\mathrm{CN}+/-$ (both $p<0.01)$ groups, and the count was also higher in the $\mathrm{MCI}+$ group than those in the MCI- $(p=0.019)$ and $\mathrm{CN}-(p=0.002)$ groups. Baseline peripheral NLR level was significantly higher in the $\mathrm{AD}+$ group than in the $\mathrm{MCI}+/-$ (both $p<0.05$ ), and $\mathrm{CN}+/-$ (both $p<0.01$ ) groups. The level was also higher in the MCI+ group compared with those in the MCI$(p=0.001)$ and $\mathrm{CN}-(p<0.001)$ groups. And the level in $\mathrm{CN}+$ group is higher than that in $\mathrm{CN}-(p=0.020)$ group. Baseline peripheral LYM count was significantly 
Table 1 Basic characteristics of population included

\begin{tabular}{|c|c|c|c|c|c|c|}
\hline Characteristics & $\mathrm{CN}-$ & $\mathrm{CN}+$ & $\mathrm{MCl}-$ & $\mathrm{MCl}+$ & $A D+$ & $p$ \\
\hline Number & 168 & 123 & 217 & 403 & 196 & \\
\hline Age (years) & $73.41 \pm 5.82$ & $75.34 \pm 5.89$ & $70.78 \pm 8.01$ & $73.21 \pm 7.14$ & $74.35 \pm 8.00$ & $<0.001$ \\
\hline Female gender (\%) & $81(48.21)$ & $65(52.85)$ & $94(43.32)$ & $163(40.45)$ & $83(42.35)$ & 0.114 \\
\hline Education (years) & $16.40 \pm 2.65$ & $16.17 \pm 2.66$ & $16.15 \pm 2.69$ & $16.01 \pm 2.81$ & $15.47 \pm 2.97$ & 0.042 \\
\hline APOE \&4 carriers (\%) & $22(13.10)$ & $54(43.90)$ & $44(20.28)$ & $263(65.26)$ & $148(75.51)$ & $<0.001$ \\
\hline \multicolumn{7}{|l|}{ Biomarkers } \\
\hline CSF A $\beta(p g / m l)$ & $237.65 \pm 25.07$ & $147.55 \pm 25.88$ & $234.05 \pm 25.86$ & $138.18 \pm 24.93$ & $129.95 \pm 22.01$ & $<0.001$ \\
\hline CSF P-tau (pg/ml) & $26.27 \pm 12.25$ & $35.48 \pm 18.43$ & $24.81 \pm 11.64$ & $45.09 \pm 19.58$ & $47.02 \pm 18.95$ & $<0.001$ \\
\hline CSF T-tau (pg/ml) & $59.76 \pm 23.74$ & $75.96 \pm 37.86$ & $57.00 \pm 25.10$ & $103.7 \pm 48.62$ & $122.39 \pm 49.86$ & $<0.001$ \\
\hline FDG-PET & $1.33 \pm 0.12$ & $1.30 \pm 0.13$ & $1.30 \pm 0.11$ & $1.22 \pm 0.13$ & $1.06 \pm 0.13$ & $<0.001$ \\
\hline \multicolumn{7}{|l|}{ Cognitive scores } \\
\hline MMSE & $29.01 \pm 1.25$ & $29.04 \pm 1.14$ & $28.27 \pm 1.60$ & $27.41 \pm 1.86$ & $23.24 \pm 1.95$ & $<0.001$ \\
\hline CDRSB & $0.03 \pm 0.12$ & $0.03 \pm 0.14$ & $1.25 \pm 0.76$ & $1.61 \pm 0.92$ & $4.49 \pm 1.60$ & $<0.001$ \\
\hline ADAS & $9.09 \pm 4.38$ & $9.74 \pm 4.38$ & $12.93 \pm 5.84$ & $17.41 \pm 6.79$ & $30.43 \pm 8.20$ & $<0.001$ \\
\hline MEM & $1.16 \pm 0.53$ & $0.95+0.59$ & $0.48 \pm 0.69$ & $-0.06 \pm 0.56$ & $-0.87 \pm 0.50$ & $<0.001$ \\
\hline $\mathrm{EF}$ & $0.94 \pm 0.79$ & $0.54 \pm 0.73$ & $0.53 \pm 0.89$ & $0.01 \pm 0.82$ & $-0.91 \pm 0.87$ & $<0.001$ \\
\hline \multicolumn{7}{|l|}{ Brain structure } \\
\hline $\mathrm{HV}\left(\mathrm{mm}^{3}\right)$ & $7446.36 \pm 908.34$ & $7363.18 \pm 785.76$ & $7293.58 \pm 1154.07$ & $6633.39 \pm 1058.85$ & $5854.03 \pm 1013.51$ & $<0.001$ \\
\hline EC thickness (mm) & $3845.57 \pm 635.32$ & $3855.90 \pm 547.01$ & $3737.91 \pm 684.43$ & $3454.81 \pm 733.31$ & $2833.39 \pm 661.89$ & $<0.001$ \\
\hline $\begin{array}{l}\text { Ventricular volume } \\
\left(\mathrm{mm}^{3}\right)\end{array}$ & $30620.87 \pm 15303.06$ & $37625.13 \pm 19034.18$ & $34734.47 \pm 19635.79$ & $40983.89 \pm 21601.68$ & $47828.71 \pm 21097.12$ & $<0.001$ \\
\hline \multicolumn{7}{|l|}{ Peripheral immune cells } \\
\hline $\operatorname{NEU}\left(\times 10^{3} / \mu \mathrm{L}\right)$ & $3.77 \pm 1.89$ & $4.01 \pm 1.17$ & $3.87 \pm 1.08$ & $4.10 \pm 1.19$ & $4.28 \pm 1.20$ & $<0.001$ \\
\hline $\operatorname{LYM}\left(\times 10^{3} / \mu \mathrm{L}\right)$ & $1.89 \pm 0.53$ & $1.74 \pm 0.47$ & $1.87 \pm 0.58$ & $1.71 \pm 0.53$ & $1.70 \pm 0.52$ & $<0.001$ \\
\hline $\operatorname{NLR}\left(\times 10^{3} / \mu \mathrm{L}\right)$ & $2.14 \pm 0.93$ & $2.41 \pm 0.89$ & $2.23 \pm 0.92$ & $2.50 \pm 0.96$ & $2.71 \pm 1.06$ & $<0.001$ \\
\hline
\end{tabular}

Abbreviations: $C N$ cognitively normal, $M C l$ mild cognitive impairment, $A D$ Alzheimer's disease, $C S F$ cerebrospinal fluid, $A \beta \beta$-amyloid, $P$-tau phosphorylatedtau, T-tau total tau, FDG-PET 18F-fluorodeoxyglucose-positron emission tomography, MMSE Mini-Mental State, CDRSB Clinical Dementia Rating Sum of Boxes, ADAS Alzheimer's Disease Assessment Scale, MEM memory function, EF executive function, $H V$ hippocampal volume, $E C$ entorhinal cortex, $N E U$ neutrophils, LYM lymphocytes, NLR neutrophil-lymphocyte ratio; values are mean \pm standard deviation (SD), or $n$ (\% of the group)

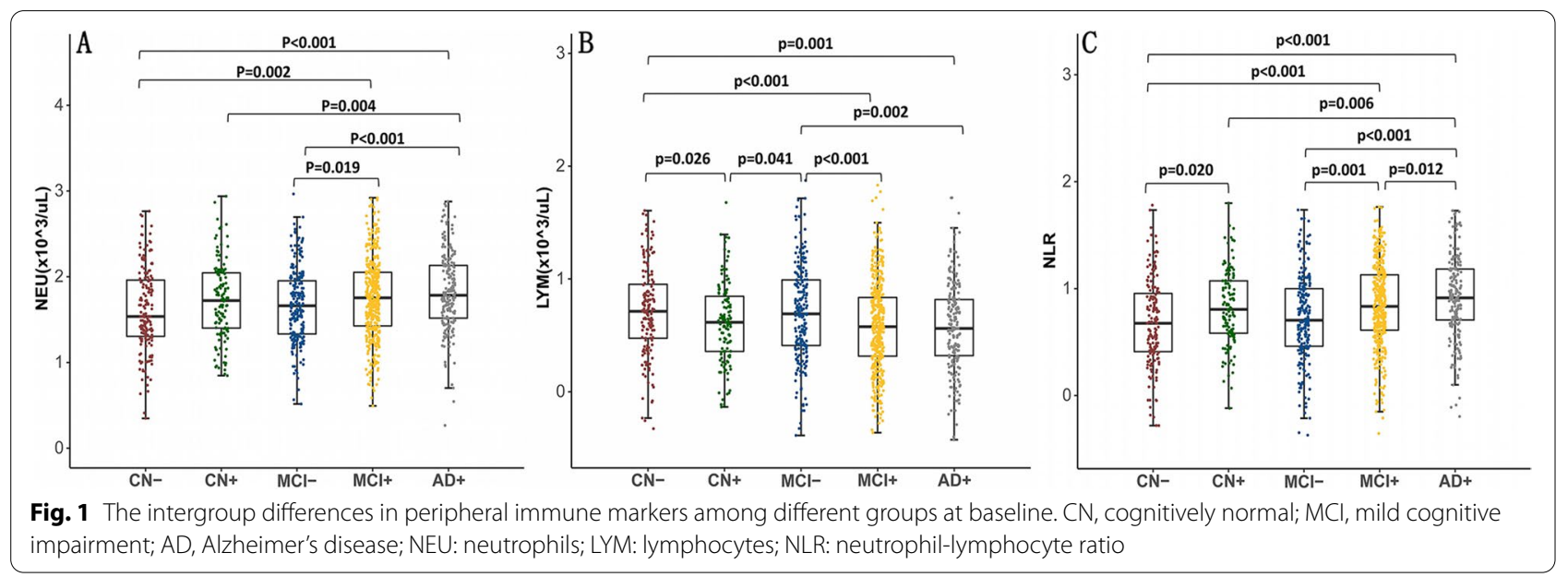

lower in the $\mathrm{AD}+$ group than those in $\mathrm{MCI}-(p=0.002)$ and $\mathrm{CN}-(p=0.001)$ groups. The count was also lower in the MCI+ group compared with $\mathrm{MCI}-(p<0.001)$ and $\mathrm{CN}-(p<0.001)$ groups. The count in $\mathrm{CN}-$ group is lower than in $\mathrm{CN}+(p=0.026)$ group, and the count in $\mathrm{MCI}-$ is lower than that in $\mathrm{CN}+(p=0.041)$ group. 


\section{Baseline associations of peripheral immunity} with cognition, neuroimaging, and AD pathology

Without considering diagnostic category, findings in the general population at baseline were shown in Fig. 2A and Additional file 2. Elevated NEU count was associated with reduced brain metabolism $(\beta=-0.035, p=0.006$ for FDGPET), lower levels of global cognition $(\beta=0.121, p<0.001$ for CDRSB, $\beta=0.172, p=0.041$ for ADAS), MEM $(\beta=$ $-0.177, p=0.012)$ and $\operatorname{EF}(\beta=-0.231, p=0.006)$, as well as greater ventricular volume $(\beta=0.416, p=0.042)$. Elevated level of NLR was associated with a lower level of $\mathrm{A} \beta(\beta$ $=-0.078, p<0.001)$ and a higher level of T-tau $(\beta=0.080$, $p=0.033)$ in CSF, reduced brain metabolism $(\beta=-0.050$, $p=0.001$ for FDG-PET), lower levels of global cognition $(\beta$ $=0.153, p<0.001$ for CDRSB, and $\beta=0.391, p<0.001$ for ADAS), MEM $(\beta=-0.322, p<0.001)$ and $\operatorname{EF}(\beta=-0.306$, $p=0.002)$, smaller hippocampal volume (HV) $(\beta=-0.184$, $p=0.011)$, and lesser entorhinal cortex (EC) thickness $(\beta=$ $-0.217, p=0.004)$, as well as greater ventricular volume $(\beta$ $=0.889, p<0.001)$. However, elevated LYM count was associated with a higher level of $\mathrm{A} \beta(\beta=0.071, p<0.001)$ and a lower level of T-tau $(\beta=-0.124, p=0.001)$ in CSF, better levels of global cognition $(\beta=-0.066 p=0.051$ for CDRSB, and $\beta=-0.263, p=0.007$ for ADAS $)$ and MEM $(\beta=0.198$, $p=0.018)$, greater $\mathrm{HV}(\beta=0.200, p=0.006)$ and EC thickness $(\beta=0.185, p=0.014)$, as well as smaller ventricular volume $(\beta=-0.652, p=0.006)$.
Taking diagnostic categories into consideration barely changed the identified associations of peripheral immunity with cognition, neuroimaging, and $\mathrm{AD}$ pathology (see Additional files 4, 5 and 6).

\section{Associations of baseline peripheral immunity with longitudinal changes in cognition, neuroimaging, and AD pathology}

Regardless of diagnosis category, as shown in Fig. 2B-D and Additional file 3, higher NEU count and higher NLR level were associated with a faster cognitive decline as indicated by changes in MMSE score (estimate $=-0.037, p=0.005$ for NEU, and estimate $=$ $-0.081, p<0.001$ for NLR), CDRSB score (estimate $=0.023, p<0.001$ for NEU, and estimate $=0.038, p$ $<0.001$ for NLR), ADAS score (estimate $=0.027, p=$ 0.032 for NEU, and estimate $=0.047, p=0.001$ for NLR), MEM score (estimate $=-0.036, p=0.003$ for NEU, and estimate $=-0.043, p=0.003$ for NLR), EF score (estimate $=-0.057, p=0.011$ for NEU, and estimate $=-0.055, p=0.034$ for NLR), and ventricular volume (estimate $=0.034, p=0.032$ for NEU, and estimate $=0.057, p=0.002$ for NLR), higher NLR level was associated with a faster cognitive decline as indicated by changes in HV (estimate $=-0.030, p=$ 0.002). Besides, higher LYM count was associated with a slower cognitive decline as indicated by changes in

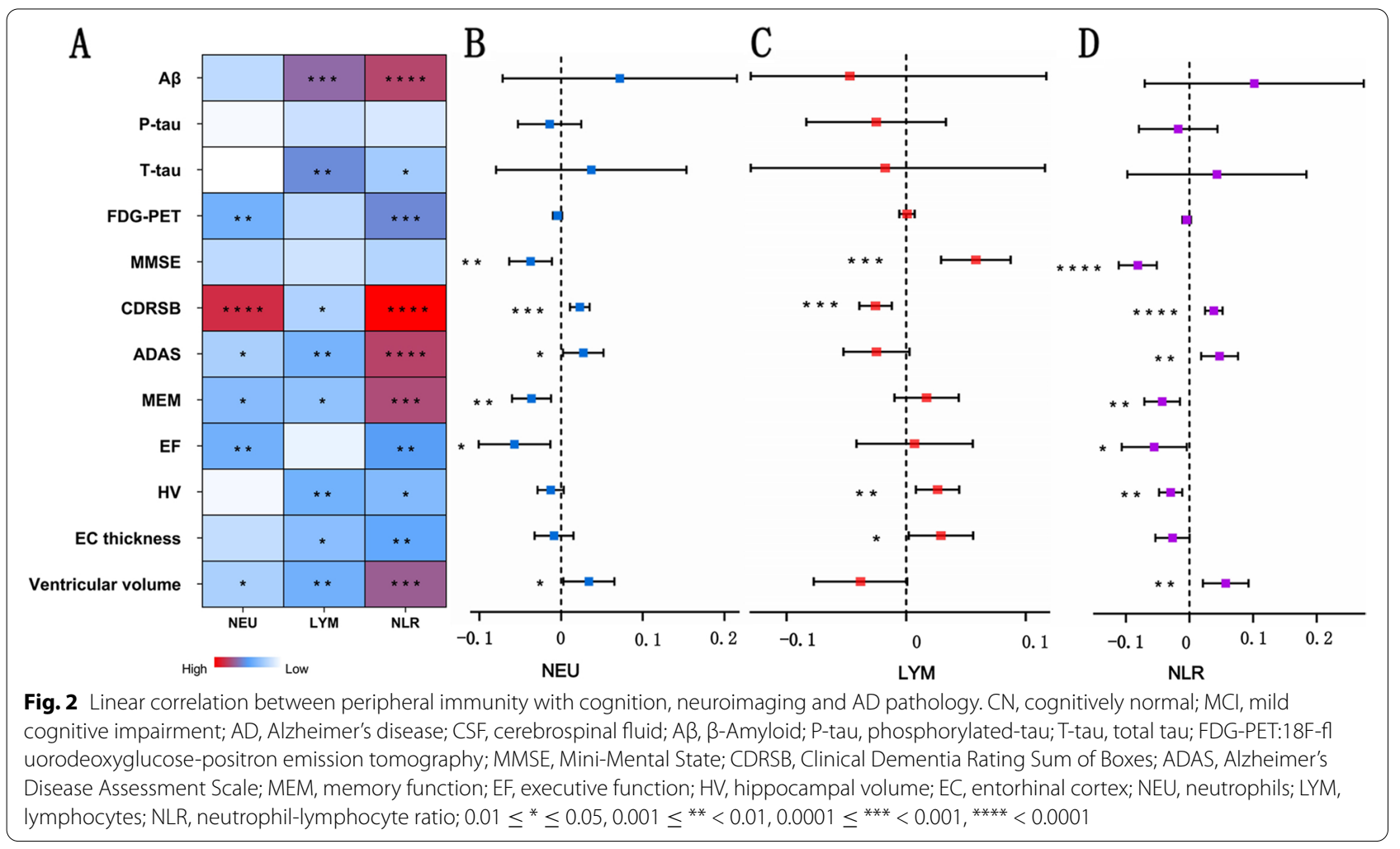


MMSE score (estimate $=0.058, p<0.001$ ), CDRSB score (estimate $=-0.026, p<0.001$ ), HV (estimate $=$ 0.026, $p=0.005$ ), and EC thickness (estimate $=0.029$, $p=0.036)$.

Moreover, taking diagnostic categories into consideration, statistically significant longitudinal correlations were still found between peripheral immunity with cognition, neuroimaging, and AD pathology in each group (see Additional files 7, 8 and 9). The directions of the above longitudinal correlations were consistent with the directions of the cross-sectional correlations, indicated by regression coefficients.

\section{Interaction analyses}

We found that the interaction between NLR and gender was obviously associated with $\mathrm{HV}(p=0.024)$. Subgroup analyses showed that significant associations between NLR and HV were found only in female subgroup $(\beta=-0.346, p<0.001)$, but not in male subgroup $(\beta=-0.033, p=0.740)$. No interaction was found of peripheral immune markers with $A P O E \varepsilon 4$ status (see Additional file 10).

\section{Causal mediation analyses}

The cross-sectional analysis indicated that CSF A $\beta$ and T-tau were not only important biomarkers for cognitive impairment but also potential modulators of cognition. The indirect and total effects of LYM count on cognition, including global cognition measured by ADAS (Fig. 3A, D) as well as MEM (Fig. 3B, E), and EF (Fig. 3C, F) reached statistical significance $(p<0.05)$, but the direct effects did not $(p>0.05)$, indicating that all the associations of LYM with global cognition, MEM, and EF were completely mediated by $A \beta$ and T-tau, with the ratio of mediation ranging from 38 to $64 \%$. The total effects of LYM count on EF did not reach statistical significance, which might be owing to the masking effect [13]. The direct effects of NLR on cognition, including global cognition (Fig. 3D, J), MEM (Fig. 3E, K) and EF (Fig. 3E, L), were significantly lower than the indirect effects, indicating all the associations of NLR with MEM, EF and global cognition were partially mediated by $\mathrm{A} \beta$ and $\mathrm{T}$-tau, with the ratio of mediation ranging from 18 to $39 \%$.

\section{Discussion}

This is a timely study that systematically explored the associations of peripheral immunity with cognition, neuroimaging and $\mathrm{AD}$ pathology, and explored the mediation effects of AD pathology on cognition. Precisely, our study showed two types of peripheral immune cells (NEU and LYM), and the ratio of these two cell types (NLR) were associated with AD pathology (CSF A $\beta$, T-tau), brain metabolism (FDG-PET), global cognition (MMSE,
CDRSB and ADAS scores), MEM, EF, and neuroimaging of AD (HV, EC thickness and ventricular volume) at baseline and follow-up, and the pathological effects of these peripheral immune markers were different. Furthermore, the associations among peripheral immunity and $\mathrm{AD}$ pathology, cognition, and neuroimaging were independent of $A P O E \varepsilon 4$ status, and $\mathrm{A} \beta$ and T-tau pathology could mediate the influences of LYM count and NLR on cognition.

Although the detailed roles the immune system plays in $\mathrm{AD}$ are not fully understood and controversial, existing studies have shown a direct communication between the peripheral and central immune system [4, 14], and the changes observed in the peripheral blood are a reflection of the immune response in the brain mediated by proinflammatory cytokines that are released to the periphery. A recent study showed that a history of infections requiring hospitalization was associated with future development of $\mathrm{AD}$ [15], and existing meta-analysis found that non-steroidal anti-inflammatory drug users had a lower risk of developing AD compared with nonusers [16].

Neurofibrillary tangles and widespread senile plaques in the AD brain provide a significant stimulus for inflammation. Increased serum levels of tumor necrosis factor (TNF), ICAM-1, IL-1, IL-6, and other already reported pro-inflammatory cytokines in $\mathrm{AD}$ patients [17-22] can cause a neutrophil "alert" activation status, which is reflected by a significantly increased of NEU CD11b and Mac-1 in blood [23]. Tiffany et al. discovered that A $\beta$ was a formyl-peptide receptor 2 agonist, indicating that $A \beta$ was a potent chemoattractant for phagocytic leukocytes [24]. Proinflammatory cytokines contribute to neuronal dysfunction and cell death [25], and those released to the periphery can further cause changes in peripheral NEU count. Neurovasculature not only allows NEU to enter the central nervous system (CNS) but also promotes the accumulation of NEU in the CNS during neuroinflammation [26]. Once NEU are in proximity of $A \beta$ plaques, they are activated and subsequently secrete detrimental mediators, including reactive oxygen species (ROS) [27], which further increases the permeability of the blood brain barrier (BBB) by damaging tight junctions [28]. Joseph Park et al. found that NEU were involved in $\mathrm{A} \beta$-activated microglia-mediated AD pathology to enhance neuroinflammation [29]. Increased senescent NEU may act through enhanced tissue tropism and the damaged BBB to migrate to the amyloid plaque sites and release NETs, leading to aggravation of $A \beta$ pathology $[18,26,30]$. Therefore, a vicious positive feedback cycle that can explain the associations between NEU and $\mathrm{AD}$ is proposed: upregulated senescent NEU that overexpress CD83 and TAP1 stimulate T cells by antigen presentation, and activated $\mathrm{T}$ cells in turn release 


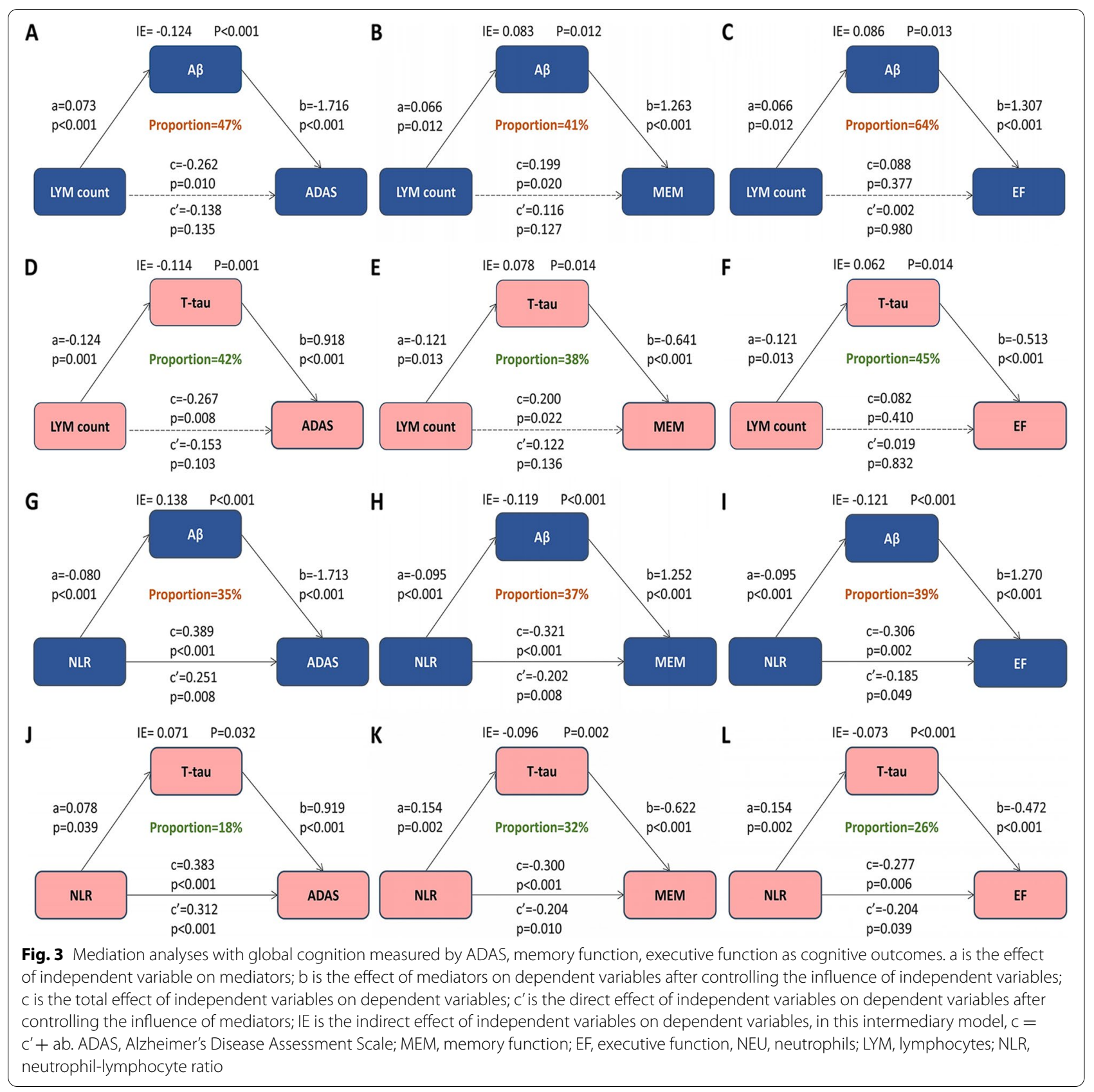

proinflammatory markers which elevate the number of senescent NEU [26]. Yuan Dong et al. found that NEU phenotype might be associated with the rate of cognitive decline [28]. Kritleen $\mathrm{K}$ et al. found that NEU-related inflammatory factor could predict the decline in EF [31]. All the above evidences suggest that increased count of peripheral NEU has an adverse effect on cognitive function, which is consistent with our results.

With respect to LYM, the issue becomes even more complicated because there is currently no consensus on the modifications of LYM in AD. Our results are consistent with several studies that reported a reduction in the number of peripheral LYM in AD patients [32-37], which supports the hypothesis of a general decline in immune activity and cell cycle dysregulation in AD that creates a permissive environment to the pathophysiological processes happening in the brain. Cell cycle dysregulation is systemic, affecting not only neurons [38] but also peripheral LYM. Adaptive immune cells including $\mathrm{T}$ and B lymphocytes play a major part in the inflammatory 
reaction in the brains of the $\mathrm{AD}$ patients. Compared with healthy controls, differentiated CD3+ T-cells is increased in AD hippocampal parenchyma [6], and activated $\mathrm{T}$ cells generate interferon gamma (IFN- $\alpha$ ) that can result in the deposition of $A \beta$, cognitive impairment, and subsequently $\mathrm{AD}$ [39]. During the inflammatory response, $\mathrm{BBB}$ is destroyed in the brains of AD patients, LYM in the peripheral blood migrate to and infiltrate into the brain, and LYM count number increases especially in the hippocampus and temporal cortex [40]. Microglia will recruit LYM from the peripheral circulation across the broken BBB into the CNS by releasing TNF- $\alpha$, thus decreasing the amount of LYM in the peripheral circulation [41]. LYM in the peripheral blood migrate to the brain, infiltrate into the brain tissue [40], and facilitate the interaction between the CNS and immune system [42]. Gate et al. detected CD8+ T cells in AD-affected hippocampus and found that $\mathrm{CD} 8+\mathrm{T}$ cells were adjacent to $A \beta$ plaques [43]. In AD patients, LYM are more sensitive to ROS than in healthy controls [44]. As was mentioned, the increase of NEU count in patients with $\mathrm{AD}$ could lead to an increase in the release of ROS, which might be part of the reason for the observed decrease in peripheral LYM. Moreover, specific mutations relevant to AD including presenilin 1 mutation might cause the circulating LYM in AD brains more susceptible to cell death [45]. All the above evidence showed an inverse correlation between the increased count of peripheral LYM and AD diagnosis.

Moreover, we discovered that the ratio of the above two types of peripheral immune cells known as NLR was also an adverse indicator for $\mathrm{AD}$, which has also been used in several other disorders, such as cardiovascular disease [46], diabetes [47], colorectal cancer [48], lung cancer [49], and Parkinson's disease [37, 50]. Our study was consistent with these previous studies which observed a dramatic increase of peripheral NLR in AD patients compared to healthy controls [7, 51], suggesting that higher peripheral NLR has an adverse effect on cognitive function.

Prior studies showed that $A \beta$ might be sufficient to cause cortical amyloid deposition [52] and neurodegeneration which ultimately led to cognitive deterioration [53], while T-tau probably was associated with the intensity of neuronal injury [54] and neurodegeneration [52]. Both of them had high accuracy in AD diagnosis. Our mediation analysis indicated that $\mathrm{A} \beta$ and T-tau might fully mediate the influences of NLR on global cognition, MEM, and EF, while $A \beta$ and T-tau might partially mediate the influences of LYM on global cognition, MEM, and EF. Thus, it could be reasonably inferred that LYM count and NLR led to AD by contributing to cortical amyloid deposition and neuronal injury, or the causal relationship between LYM count and NLR with A $\beta$ and T-tau might be bidirectional. These findings indicated the close associations of LYM count and NLR with cognition.

Historically, CSF and imaging biomarkers have been considered as the indicators tightly related to AD. However, the invasiveness of lumbar punctures and the cost of imaging examination limited their application. Blood-based biomarkers such as peripheral immune cells have advantages of simple operation, low cost, easy acceptability, and potential global applicability [55]. Thus, it is necessary to explore the associations of peripheral immune markers with cognition, CSF, and imaging biomarkers of $\mathrm{AD}$.

According to our understanding, the topic about the association between peripheral immunity and $A D$ is still in its infancy. Previous articles have investigated the association of peripheral immunity with $A D$, but they were limited to basic research $[5,6]$, animal research $[18$, 19, 30], review [26, 40, 42], and rough cross-sectional analyses [7], and most studies were of small samples [28, $33,36,44,51,56,57]$. Our study is the first original study using linear models to systematically examine the linear regression associations of peripheral immunity with cognition, neuroimaging, and $\mathrm{AD}$ pathology in a huge human cohort, the first study to explore whether the associations of peripheral immunity with cognition were mediated by AD core pathology, and the first study to explore the interaction among peripheral immunity and $A P O E \varepsilon 4$ status and gender.

In our study, patients with established AD displayed differences in NEU count, LYM count, and NLR level compared to $\mathrm{MCI}$ and $\mathrm{CN}$ groups. These intergroup differences as well as significant associations found in cross-sectional analysis indicated a positive correlation between the diagnosis of $\mathrm{AD}$ and higher peripheral NEU count and NLR level, while an inverse correlation between higher peripheral LYM counts and AD diagnosis. Longitudinal analysis indicated that higher peripheral NEU count and NLR level were associated with a faster cognitive decline, while higher peripheral LYM count was associated with a slower cognitive decline, which is specifically manifested in the changes of cognition scores and neuroimaging and Alzheimer's pathology. $A \beta$ and $T$-tau are tightly related to $A D$, and the mediating effect in this study suggested that $A \beta$ and T-tau also acted as mediators of cognition. Therefore, peripheral LYM and NLR may affect AD through cerebral amyloid deposition and neuronal injury, or the causal relationship between peripheral immune markers (LYM count and NLR) with cerebral amyloid deposition and neuronal injury might be bidirectional, thereby raising the credibility that peripheral immunity is associated with AD. For interaction effects, no interaction between peripheral immunity and $A P O E$ \&4 status was found in this study, which showed 
associations among peripheral immunity and AD pathology, cognition, and neuroimaging were independent of $A P O E \varepsilon 4$ status. However, gender strata effects existed between NLR and HV, and significant associations were only found in female subgroup, not in male subgroup, which showed the association between NLR and HV was dependent on gender. This may be because testosterone in males exerted its influence to an extent that HV was genetically and environmentally affected [36]. In summary, we found that peripheral immunity was associated with cognition, neuroimaging, and Alzheimer's pathology, which can provide ideas and support for future research in this field. If the associations can be replicated in more human cohorts in the future, blood-based biomarkers may replace CSF and imaging biomarkers in the future, and anti-inflammatory therapy may become a new direction in the treatment and prevention of AD.

\section{Limitations}

There are limitations in this study. First, our study used NEU, LYM, and NLR as indicators to reflect peripheral immune system, and other peripheral immune markers except the three should be included in future research since peripheral immune system is an intricate system. Second, the generalizability of our consequences might be limited by the study populations sources from ADNI, no longitudinal data of peripheral immune markers (NEU, LYM, and NLR) and lymphocyte subpopulations (T/B lymphocytes) were found in the ADNI cohort, which requires large-scale studies with high-quality to further analyze longitudinal correlation in the future. Third, we did not discuss the direct associations between peripheral immune markers and inflammatory markers in the nervous system. In the future, we can explore whether neuroinflammation plays a mediating role in cognition.

\section{Conclusions}

In summary, our study found that two types of peripheral immune cells (NEU and LYM) and the ratio of these two cell types (NLR) were associated with cognition, neuroimaging, and $\mathrm{AD}$ pathology; the associations of LYM count and NLR level with cognition were mediated by AD core pathology. Peripheral immune markers may replace CSF and imaging biomarkers and may provide a measure for initial screening and provide new insights into the prevention and treatment of AD.

\footnotetext{
Abbreviations

AD: Alzheimer's disease; ADNI: Alzheimer's Disease Neuroimaging Initiative; CN: Cognitively normal; MCl: Mild cognitive impairment; CSF: Cerebrospinal fluid; A $\beta$ : $\beta$-Amyloid; P-tau: Phosphorylated-tau; T-tau: Total tau; FDG-PET: 18F-fluorodeoxyglucose-positron emission tomography; MMSE: Mini-Mental
}

State; CDRSB: Clinical Dementia Rating Sum of Boxes; ADAS: Alzheimer's Disease Assessment Scale; MEM: Memory function; EF: Executive function; HV: Hippocampal volume; EC: Entorhinal cortex; NEU: Neutrophils; LYM: Lymphocytes; NLR: Neutrophil-Iymphocyte ratio; CNS: Central nervous system; SD: Standard deviation; MRI: Magnetic resonance imaging; TNF:Tumor necrosis factor; ROS: Reactive oxygen species; BBB: Blood brain barrier.

\section{Supplementary Information}

The online version contains supplementary material available at https://doi. org/10.1186/s13195-022-00968-y.

Additional file 1. Study flow diagram.

Additional file 2. Cross-sectional associations of peripheral immunity with cognition, neuroimaging and AD pathology in all participants.

Additional file 3. Longitudinal associations of peripheral immunity with cognition, neuroimaging and AD pathology in all participants.

Additional file 4. Cross-sectional associations of peripheral immunity with cognition, neuroimaging and AD pathology in CN group.

Additional file 5. Cross-sectional associations of peripheral immunity with cognition, neuroimaging and $\mathrm{AD}$ pathology in $\mathrm{MCl}$ group.

Additional file 6. Cross-sectional associations of peripheral immunity with cognition, neuroimaging and $A D$ pathology in $A D$ group.

Additional file 7. Longitudinal associations of peripheral immunity with cognition, neuroimaging and AD pathology in CN group.

Additional file 8. Longitudinal associations of peripheral immunity with cognition, neuroimaging and $\mathrm{AD}$ pathology in $\mathrm{MCl}$ group.

Additional file 9. Longitudinal associations of peripheral immunity with cognition, neuroimaging and AD pathology in AD group.

Additional file 10. Interaction analysis.

\section{Acknowledgements}

Data collection and sharing for this project was funded by the Alzheimer's Disease Neuroimaging Initiative (ADNI) (National Institutes of Health Grant U01 AG024904) and DOD ADNI (Department of Defense award number W81XWH-12-2-0012). ADNI is funded by the National Institute on Aging, the National Institute of Biomedical Imaging and Bioengineering, and through generous contributions from the following: AbbVie, Alzheimer's Association; Alzheimer's Drug Discovery Foundation; Araclon Biotech; BioClinica, Inc.;Biogen; Bristol-Myers Squibb Company; CereSpir, Inc.; Cogstate; Eisai Inc.; Elan Pharmaceuticals, Inc.; Eli Lilly and Company; Eurolmmun; F. Hoffmann-La Roche Ltd. and its affiliated company Genentech, Inc.; Fujirebio; GE Healthcare; IXICO Ltd.; Janssen Alzheimer Immunotherapy Research \& Development, LLC.; Johnson \& Johnson Pharmaceutical Research \& Development LLC.; Lumosity; Lundbeck; Merck \& Co., Inc.; Meso Scale Diagnostics, LLC.; NeuroRx Research; Neurotrack Technologies; Novartis Pharmaceuticals Corporation; Pfizer Inc.; Piramal Imaging; Servier; Takeda Pharmaceutical Company; and Transition Therapeutics. The Canadian Institutes of Health Research is providing funds to support ADNI clinical sites in Canada. Private sector contributions are facilitated by the Foundation for the National Institutes of Health (www.fnih. org). The grantee organization is the Northern California Institute for Research and education, and the study is coordinated by the Alzheimer's Therapeutic Research Institute at the University of Southern California. ADNI data are disseminated by the Laboratory for Neuro Imaging at the University of Southern California. Data used in the preparation of this article were obtained from the Alzheimer's Disease Neuroimaging Initiative (ADNI) database (adni.loni.usc. edu). As such, the investigators within the ADNI contributed to the design and implementation of ADNI and/or provided data but did not participate in analysis or writing of this report. A complete listing of ADNI investigators can be found at: http://adni.loni.usc.edu/wp-content/uploads/how_to_ apply/ADNI_Acknowledgement_List.pdf.

\section{Authors' contributions}

JTY conceptualized the study and revised the manuscript. JHH and YNO analyzed and interpreted the data, drafted and revised the manuscript, did 
the statistical analysis, and prepared all the figures. WX and PFZ participated in the interpretation of the data and revision of the manuscript. All authors contributed to the writing and revisions of the paper and approved the final version. Data used in the preparation of this article were obtained from the Alzheimer's Disease Neuroimaging Initiative (ADNI) database (adni.loni.usc edu). As such, the investigators within the ADNI contributed to the design and implementation of ADNI and/or provided data but did not participate in analysis or writing of this report.

\section{Funding}

This study was supported by grants from grants from the National Natural Science Foundation of China $(82071201$, 91849126), the National Key R\&D Program of China (2018YFC1314702), Shanghai Municipal Science and Technology Major Project (No.2018SHZDZX01) and ZHANGJIANG LAB, Tianqiao and Chrissy Chen Institute, and the State Key Laboratory of Neurobiology and Frontiers Center for Brain Science of Ministry of Education, Fudan University.

\section{Availability of data and materials}

The dataset generated and analyzed in the current study is available from the corresponding author on reasonable request.

\section{Declarations}

\section{Ethics approval and consent to participate}

The study was approved by institutional review boards of all participating institutions, and written informed consent was obtained from all participants or their guardians according to the Declaration of Helsinki (consent for research).

\section{Consent for publication}

Not applicable.

\section{Competing interests}

The authors declare that they have no competing interests.

Received: 7 November 2021 Accepted: 30 January 2022

Published online: 09 February 2022

\section{References}

1. Reiman EM, et al. Alzheimer's disease: implications of the updated diagnostic and research criteria. J Clin Psychiatry. 2011;72(9):1190-6.

2. Mattsson $\mathrm{N}$, et al. Association of plasma neurofilament light with neurodegeneration in patients with Alzheimer disease. JAMA Neurol. 2017:74(5):557-66.

3. Casserly IP, Topol EJ. Convergence of atherosclerosis and alzheimer's disease: cholesterol, inflammation, and misfolded proteins. Discov Med. 2004;4(22):149-56.

4. Walker KA, Ficek BN, Westbrook R. Understanding the role of systemic inflammation in Alzheimer's disease. ACS Chem Neurosci. 2019;10(8):3340-2.

5. Scali C, et al. Neutrophils CD11b and fibroblasts PGE(2) are elevated in Alzheimer's disease. Neurobiol Aging. 2002;23(4):523-30.

6. Togo T, et al. Occurrence of T cells in the brain of Alzheimer's disease and other neurological diseases. J Neuroimmunol. 2002;124(1-2):83-92.

7. Kuyumcu ME, et al. The evaluation of neutrophil-lymphocyte ratio in Alzheimer's disease. Dement Geriatr Cogn Disord. 2012;34(2):69-74.

8. Wyss-Coray T. Inflammation in Alzheimer disease: driving force, bystander or beneficial response? Nat Med. 2006;12(9):1005-15.

9. Gibbons LE, et al. A composite score for executive functioning, validated in Alzheimer's Disease Neuroimaging Initiative (ADNI) participants with baseline mild cognitive impairment. Brain Imaging Behav. 2012;6(4):517-27.

10. Crane PK, et al. Development and assessment of a composite score for memory in the Alzheimer's Disease Neuroimaging Initiative (ADNI). Brain Imaging Behav. 2012;6(4):502-16.

11. Lowe VJ, et al. Application of the National Institute on Aging-Alzheimer's Association AD criteria to ADNI. Neurology. 2013;80(23):2130-7.
12. Baron RM, Kenny DA. The moderator-mediator variable distinction in social psychological research: conceptual, strategic, and statistical considerations. J Pers Soc Psychol. 1986;51(6):1173-82.

13. MacKinnon DP, Krull JL, Lockwood CM. Equivalence of the mediation, confounding and suppression effect. Prev Sci. 2000;1(4):173-81.

14. Bettcher BM, et al. Peripheral and central immune system crosstalk in Alzheimer disease - a research prospectus. Nat Rev Neurol. 2021;17(11):689-701.

15. Sipilä PN, et al. Hospital-treated infectious diseases and the risk of dementia: a large, multicohort, observational study with a replication cohort. Lancet Infect Dis. 2021;21(11):1557-67.

16. Wang J, et al. Anti-inflammatory drugs and risk of Alzheimer's disease: an updated systematic review and meta-analysis. J Alzheimers Dis. 2015;44(2):385-96

17. Singh VK. Neuroautoimmunity: pathogenic implications for Alzheimer's disease. Gerontology. 1997;43(1-2):79-94.

18. Baik SH, et al. Migration of neutrophils targeting amyloid plaques in Alzheimer's disease mouse model. Neurobiol Aging. 2014;35(6):1286-92.

19. Zenaro E, et al. Neutrophils promote Alzheimer's disease-like pathology and cognitive decline via LFA-1 integrin. Nat Med. 2015;21(8):880-6.

20. Pellicanò $M$, et al. Systemic immune responses in Alzheimer's disease: in vitro mononuclear cell activation and cytokine production. J Alzheimers Dis. 2010;21(1):181-92.

21. Griffin WS, Mrak RE. Interleukin-1 in the genesis and progression of and risk for development of neuronal degeneration in Alzheimer's disease. J Leukoc Biol. 2002;72(2):233-8.

22. Cacquevel M, et al. Cytokines in neuroinflammation and Alzheimer's disease. Curr Drug Targets. 2004;5(6):529-34.

23. Remarque EJ, et al. Patients with Alzheimer's disease display a pro-inflammatory phenotype. Exp Gerontol. 2001;36(1):171-6.

24. Bufe $B$, et al. Recognition of bacterial signal peptides by mammalian formyl peptide receptors: a new mechanism for sensing pathogens. J Biol Chem. 2015;290(12):7369-87.

25. Relton JK, Rothwell NJ. Interleukin-1 receptor antagonist inhibits ischaemic and excitotoxic neuronal damage in the rat. Brain Res Bull. 1992;29(2):243-6.

26. Sayed A, et al. The neutrophil-to-lymphocyte ratio in Alzheimer's disease: current understanding and potential applications. J Neuroimmunol. 2020;349:577398

27. Zhao Y, Zhao B. Oxidative stress and the pathogenesis of Alzheimer's disease. Oxid Med Cell Longev. 2013;2013:316523.

28. Dong $Y$, et al. Neutrophil hyperactivation correlates with Alzheimer's disease progression. Ann Neurol. 2018;83(2):387-405.

29. Park J, et al. Mimicry of central-peripheral immunity in Alzheimer's disease and discovery of neurodegenerative roles in neutrophil. Front Immunol. 2019;10:2231.

30. Pietronigro EC, et al. NETosis in Alzheimer's disease. Front Immunol. 2017:8:211.

31. Bawa KK, et al. A peripheral neutrophil-related inflammatory factor predicts a decline in executive function in mild Alzheimer's disease. J Neuroinflammation. 2020;17(1):84

32. Richartz-Salzburger $E$, et al. Altered lymphocyte distribution in Alzheimer's disease. J Psychiatr Res. 2007:41(1-2):174-8.

33. Tavolato B, Argentiero V. Immunological indices in presenile Alzheimer's disease. J Neurol Sci. 1980;46(3):325-31.

34. Tollefson GD, et al. Lymphopenia in primary degenerative dementia. J Psychiatr Res. 1989;23(3-4):191-9.

35. Speciale $L$, et al. Lymphocyte subset patterns and cytokine production in Alzheimer's disease patients. Neurobiol Aging. 2007;28(8):1163-9.

36. Chen $\mathrm{SH}$, et al. Altered peripheral profile of blood cells in Alzheimer disease: a hospital-based case-control study. Medicine (Baltimore). 2017:96(21):e6843.

37. Jensen MP, et al. Lower lymphocyte count is associated with increased risk of Parkinson's disease. Ann Neurol. 2021;89(4):803-12.

38. Busser J, Geldmacher DS, Herrup K. Ectopic cell cycle proteins predict the sites of neuronal cell death in Alzheimer's disease brain. J Neurosci. 1998;18(8):2801-7.

39. Browne TC, et al. IFN- $\gamma$ production by amyloid $\beta$-specific Th1 cells promotes microglial activation and increases plaque burden in a mouse model of Alzheimer's disease. J Immunol. 2013;190(5):2241-51. 
40. Sardi F, et al. Alzheimer's disease, autoimmunity and inflammation. the good, the bad and the ugly. Autoimmun Rev. 2011;11(2):149-53.

41. Liu CC, et al. Apolipoprotein E and Alzheimer disease: risk, mechanisms and therapy. Nat Rev Neurol. 2013;9(2):106-18.

42. Britschgi M, Wyss-Coray T. Systemic and acquired immune responses in Alzheimer's disease. Int Rev Neurobiol. 2007;82:205-33.

43. Gate D, et al. Clonally expanded CD8 T cells patrol the cerebrospinal fluid in Alzheimer's disease. Nature. 2020;577(7790):399-404.

44. Ponce DP, et al. Increased susceptibility to oxidative death of lymphocytes from Alzheimer patients correlates with dementia severity. Curr Alzheimer Res. 2014;11(9):892-8.

45. Rezai-Zadeh K, et al. Can peripheral leukocytes be used as Alzheimer's disease biomarkers? Expert Rev Neurother. 2009;9(11):1623-33.

46. Bhat T, et al. Neutrophil to lymphocyte ratio and cardiovascular diseases: a review. Expert Rev Cardiovasc Ther. 2013;11(1):55-9.

47. Imtiaz F, et al. Neutrophil lymphocyte ratio as a measure of systemic inflammation in prevalent chronic diseases in Asian population. Int Arch Med. 2012;5(1):2.

48. Walsh SR, et al. Neutrophil-lymphocyte ratio as a prognostic factor in colorectal cancer. J Surg Oncol. 2005;91(3):181-4.

49. Sarraf KM, et al. Neutrophil/lymphocyte ratio and its association with survival after complete resection in non-small cell lung cancer. J Thorac Cardiovasc Surg. 2009;137(2):425-8.

50. Akil $E$, et al. The increase of carcinoembryonic antigen (CEA), high-sensitivity C-reactive protein, and neutrophil/lymphocyte ratio in Parkinson's disease. Neurol Sci. 2015;36(3):423-8.

51. Dong $X$, et al. Predictive value of routine peripheral blood biomarkers in Alzheimer's disease. Front Aging Neurosci. 2019;11:332.

52. Scheltens $P$, et al. Alzheimer's disease. The Lancet. 2016;388(10043):505-17.

53. Bennett DA, et al. Neurofibrillary tangles mediate the association of amyloid load with clinical Alzheimer disease and level of cognitive function. Arch Neurol. 2004;61(3):378-84.

54. Blennow K, Hampel H. CSF markers for incipient Alzheimer's disease. Lancet Neurol. 2003;2(10):605-13.

55. Hampel $\mathrm{H}$, et al. Blood-based biomarkers for Alzheimer disease: mapping the road to the clinic. Nat Rev Neurol. 2018;14(11):639-52.

56. Shad KF, et al. Peripheral markers of Alzheimer's disease: surveillance of white blood cells. Synapse. 2013;67(8):541-3.

57. Shalit F, et al. T lymphocyte subpopulations and activation markers correlate with severity of Alzheimer's disease. Clin Immunol Immunopathol. 1995;75(3):246-50.

\section{Publisher's Note}

Springer Nature remains neutral with regard to jurisdictional claims in published maps and institutional affiliations.

Ready to submit your research? Choose BMC and benefit from:

- fast, convenient online submission

- thorough peer review by experienced researchers in your field

- rapid publication on acceptance

- support for research data, including large and complex data types

- gold Open Access which fosters wider collaboration and increased citations

- maximum visibility for your research: over $100 \mathrm{M}$ website views per year

At BMC, research is always in progress.

Learn more biomedcentral.com/submissions 